\section{Case Reports in Oncology}

\title{
Concomitant Non-Small Cell Lung Cancer and Hairy Cell Leukemia in a Patient Harboring BRAF-V600E Mutation in Both Tissues: A Case Report
}

\author{
Maria Mitsogianni $^{\mathrm{a}}$ Nikolaos Mitsimponas $^{\mathrm{a}}$ Felipe Crespo $^{\mathrm{a}}$ \\ Karl Axel Hartmann ${ }^{b}$ Bernd Klosterhalfen ${ }^{c}$ Sabine Haase ${ }^{a}$ \\ Aristoteles Giagounidis ${ }^{a}$ \\ ${ }^{a}$ Klinik für Onkologie, Hämatologie und Palliativmedizin, Marien Hospital Düsseldorf, \\ Düsseldorf, Germany; ${ }^{b}$ Klinik für Strahlentherapie, Marien Hospital Düsseldorf, \\ Düsseldorf, Germany; ${ }^{c}$ Institut für Pathologie, Krankenhaus Düren, Düren, Germany
}

\section{Keywords}

BRAF-V600E mutation · Driver mutations · Non-small cell lung cancer · Lung adenocarcinoma - Hairy cell leukemia

\section{Abstract}

The BRAF-V600E mutation has been established as a signature alteration occurring almost universally in hairy cell leukemia. Moreover, it can be detected in a small percentage of patients with non-small cell lung cancer. We report the case of a patient with a metastatic BRAF-V600E-mutated lung adenocarcinoma suffering from concomitant hairy cell leukemia. The identification of an identical BRAF mutation in both malignancies raises physiopathological considerations and might offer unique therapeutic strategies for this group of patients. 
 Oncology}

\section{Introduction}

Lung cancer is one of the most common cancer types and the leading cause of cancerrelated deaths worldwide [1]. More than $80 \%$ of lung cancers can be classified as non-small cell lung cancer (NSCLC) [1], whereas adenocarcinoma is the most common histological subtype, accounting for $40 \%$ of all lung cancer cases [2]. In the last years, the identification of various oncogenic mutations in lung adenocarcinoma has enabled a better understanding of its pathogenesis and led to new effective targeted therapies for different molecularly defined subgroups [3,4]. One of these alterations is the mutation of the BRAF gene, which occurs in 1.6-4.9\% of NSCLC [3]. BRAF is an isoform of the RAF family of serine/threonine kinases and acts as mediator between RAS and the MAPK signaling pathway [5, 6]. BRAF activation by RAS results in a series of intracellular metabolic events regulating the cell cycle. Mutations in BRAF lead to permanent activation of the MAPK cascade and, therefore, stimulate cellular proliferation [6]. In general, BRAF has been shown to act as a driver oncogene in a wide range of malignant tumors $[7,8]$. The most commonly detected mutation consists of a valine to glutamate substitution at codon 600 of exon 15 (V600E) [7, 8]. The BRAF-V600E mutation is the predominant alteration in malignancies such as melanoma, differentiated thyroid cancer, as well as hairy cell leukemia (HCL) $[7,9,10]$. Lung adenocarcinoma, on the other hand, presents a significant heterogeneity among BRAF mutations, with approximately only $50 \%$ presenting as $\mathrm{V} 600 \mathrm{E}[7,11]$. The case reported here describes the concomitant occurrence of HCL in a patient with a BRAF-V600E-mutated lung adenocarcinoma.

\section{Case Presentation}

A 55-year-old man with a smoking history of approximately 30 pack-years was diagnosed with a tumor of the left lower lobe and underwent VATS lobectomy. Histological analysis confirmed the diagnosis of a poorly differentiated lung adenocarcinoma and further molecular diagnostics detected a mutation for BRAF-V600E. Cancer staging revealed a UICC stage Ib (pT2a, pN0, cM0) and adjuvant chemotherapy was advised but rejected by the patient.

Five months later, the patient was admitted with dysphagia, fatigue, and weight loss. Computerized tomography revealed a relapse with pulmonary, lymphatic, and peritoneal metastases as well as direct infiltration of the esophagus and pharynx. At this time, peripheral blood counts were within normal limits. Palliative systemic therapy with cisplatin, vinorelbine, and bevacizumab as well as radiation of the esophagus were initiated.

After 2 months of treatment, progressive symptomatic anemia (hemoglobin $7.4 \mathrm{~g} / \mathrm{dL}$ ) and severe neutropenia (absolute neutrophil count 270/ $\mu \mathrm{L}$ ) as well as recurrent bacterial infections were observed. The bone marrow biopsy revealed an extended infiltration by a low-grade non-Hodgkin lymphoma. Immunohistological analysis and molecular pathology detected the characteristic BRAF-V600E mutation and the diagnosis of HCL was confirmed $[12,13]$. Additional immunotherapy with rituximab was initiated, resulting in normalization of the neutrophil count and improvement of anemia.

However, in the following months, the patient developed cerebral metastasis of the NSCLC, which persisted despite radiotherapy of the neurocranium and led to death 6 months after the relapse of the disease. 
 Oncology}

\section{Discussion}

In our report, we present a patient with a BRAF-V600E-mutated lung adenocarcinoma who developed HCL after relapse of the disease. To the best of our knowledge, this is the first reported case of a patient diagnosed with a combination of these two BRAF-V600E-mutated malignancies.

A possible association of HCL with other secondary tumors such as colorectal and renal cell carcinoma was first described many years ago [14]. The identification of the BRAFV600E mutation as a somatically acquired signature alteration occurring universally in HCL $[7,9,10]$ as well as a commonly detected mutation in a series of solid tumors [7] further supported the hypothesis of a unique pathogenic mechanism in various malignant diseases. A number of patient cases with two different synchronous or metachronous BRAF-mutated malignancies have subsequently been described.

For example, a case report by Shah et al. [15] illustrated a possible connection between neuroendocrine-differentiated colorectal cancer and HCL. The patient presented by the authors was diagnosed with BRAF-V600E-mutated metastatic neuroendocrine colon cancer several years after successful treatment of HCL. Bone marrow biopsy showed no sign of a new or recurrent hematological disease and next-generation sequencing revealed no BRAFV600E mutation of the bone marrow, excluding the possibility of a germline mutation in that patient. This case confirms that somatic mutations of the BRAF gene in different cell types resulting in different malignancies can be observed in one individual, despite the rarity of such a combination.

A conclusion based on molecular testing alone is not always reliable. Schlick et al. [16] described the isolation of a BRAF-V600E mutation in a patient with NSCLC and HCL, whereas in that case immunohistochemical analysis using the monoclonal anti-BRAF-V600E (VE1) antibody revealed that the mutation detected in the lung specimen was falsely positive due to contamination with leukemic hairy cells. Although such a contamination cannot be excluded in our case, it would be unlikely, since the mutated NSCLC occurred several months before the development of any bone marrow deficiency signs.

It needs to be pointed out that the mere occurrence of two malignancies in a single patient with the same molecular aberration, be it synchronous or metachronous, does not at all imply them to be causatively linked. Independent mutations in two different histological tissues are perfectly possible and may occur at any given point in time. In the present case, a germline BRAF mutation was not ruled out. However, its presence seems unlikely given that affected patients are most often diagnosed during childhood and display distinctive facial dysmorphia, reduced growth, cardiac defects, skeletal and ectodermal anomalies as well as cognitive dysfunctions, all of which were not present in our patient.

The existence of a common pathogenic pathway as evidenced by a somatic alteration of the BRAF gene in different malignancies can offer the possibility of a unique therapeutic strategy. For example, inhibitors of the BRAF/MAPK pathway have been proven to effectively treat HCL $[17,18]$. Moreover, BRAF-targeted therapy is being widely used in the treatment of malignant melanoma [19]. In a case report by Blachly et al. [20], a patient suffering from malignant melanoma and HCL, both BRAF-V600E-mutated, achieved long-term complete remission under treatment with the BRAF-inhibitor dabrafenib. Of course, it has to be pointed out that BRAF-V600E alterations are much more common in melanoma compared to NSCLC [7]. Therefore, it can be assumed that the probability of BRAF-V600E-mutated melanoma and HCL in one individual is higher than the one of the combination described in our report. 
However, since BRAF inhibitors have been shown to be a valuable therapeutic option for patients with BRAF-mutated NSCLC [21, 22] (the combination of dabrafenib and trametinib having been approved by the FDA), efficient treatment of patients with BRAF-mutated NSCLC and HCL with a single BRAF-targeted therapy remains a realistic scenario. Further research is needed in order to detect the frequency of co-occurrence of these malignancies as well as the group of patients that could profit from a targeted personalized therapy.

\section{Statements of Ethics}

The authors have no ethical conflicts to disclose.

\section{Disclosure Statement}

The authors have no conflicts of interest to declare.

\section{References}

1 Yoon SM, Shaikh T, Hallman M. Therapeutic management options for stage III non-small cell lung cancer. World J Clin Oncol. 2017 Feb;8(1):1-20.

2 Shi J, Hua X, Zhu B, Ravichandran S, Wang M, Nguyen C et al. Somatic Genomics and Clinical Features of Lung Adenocarcinoma: A Retrospective Study. PLoS Med. 2016 Dec;13(12):e1002162.

3 Oxnard GR, Binder A, Jänne PA. New targetable oncogenes in non-small-cell lung cancer. J Clin Oncol. 2013 Mar;31(8):1097-104.

4 Park SJ, More S, Murtuza A, Woodward BD, Husain H. New Targets in Non-Small Cell Lung Cancer. Hematol Oncol Clin North Am. 2017 Feb;31(1):113-29.

5 Cantwell-Dorris ER, O'Leary JJ, Sheils OM. BRAFV600E: implications for carcinogenesis and molecular therapy. Mol Cancer Ther. 2011 Mar;10(3):385-94.

6 Cardarella S, Ogino A, Nishino M, Butaney M, Shen J, Lydon C et al. Clinical, pathologic, and biologic features associated with BRAF mutations in non-small cell lung cancer. Clin Cancer Res. 2013 Aug;19(16):4532-40.

7 Hall RD, Kudchadkar RR. BRAF mutations: signaling, epidemiology, and clinical experience in multiple malignancies. Cancer Contr. 2014 Jul;21(3):221-30.

8 Sánchez-Torres JM, Viteri S, Molina MA, Rosell R. BRAF mutant non-small cell lung cancer and treatment with BRAF inhibitors. Transl Lung Cancer Res. 2013 Jun;2(3):244-50.

9 Tiacci E, Trifonov V, Schiavoni G, Holmes A, Kern W, Martelli MP et al. BRAF mutations in hairy-cell leukemia. N Engl J Med. 2011 Jun;364(24):2305-15.

10 Arcaini L, Zibellini S, Boveri E, Riboni R, Rattotti S, Varettoni M et al. The BRAF V600E mutation in hairy cell leukemia and other mature B-cell neoplasms. Blood. 2012 Jan;119(1):188-91.

11 Cui G, Liu D, Li W, Fu X, Liang Y, Li Y et al. A meta-analysis of the association between BRAF mutation and nonsmall cell lung cancer. Medicine (Baltimore). 2017 Apr;96(14):e6552.

12 Quest GR, Johnston JB. Clinical features and diagnosis of hairy cell leukemia. Best Pract Res Clin Haematol. 2015 Dec;28(4):180-92.

13 Tiacci E, Pettirossi V, Schiavoni G, Falini B. Genomics of Hairy Cell Leukemia. J Clin Oncol. 2017 Mar; 35(9):1002-10.

14 Nielsen B, Braide I, Hasselbalch H. Evidence for an association between hairy cell leukemia and renal cell and colorectal carcinoma. Cancer. 1992 Oct;70(8):2087-90.

15 Shah H, McPherson K, Mansour A, Ramirez-Santrich C, Nassiri M, Shahda S. Neuroendocrine Differentiation of a Primary BRAF Mutant Colon Cancer in a Patient With a History of Hairy Cell Leukemia. Clin Colorectal Cancer. 2016 Dec;15(4):e235-9.

16 Schlick K, Troch M, Placher-Sorko G, Faber V, Neureiter D, Berghoff AS et al. A patient diagnosed with BRAFmutated non-small cell lung cancer and hairy cell leukemia: at last, which entity is really carrying the BRAF mutation? Ann Hematol. 2015 Feb;94(2):345-6.

17 Sarvaria A, Topp Z, Saven A. Current Therapy and New Directions in the Treatment of Hairy Cell Leukemia: A Review. JAMA Oncol. 2016 Jan;2(1):123-9. 
18 López-Rubio M, Garcia-Marco JA. Current and emerging treatment options for hairy cell leukemia. Onco Targets Ther. 2015 Aug;8:2147-56.

19 Luke JJ, Flaherty KT, Ribas A, Long GV. Targeted agents and immunotherapies: optimizing outcomes in melanoma. Nat Rev Clin Oncol. 2017 Aug;14(8):463-82.

20 Blachly JS, Lozanski G, Lucas DM, Grever MR, Kendra K, Andritsos LA. Cotreatment of hairy cell leukemia and melanoma with the BRAF inhibitor dabrafenib. J Natl Compr Canc Netw. 2015 Jan;13(1):9-13.

21 Baik CS, Myall NJ, Wakelee HA. Targeting BRAF-Mutant Non-Small Cell Lung Cancer: From Molecular Profiling to Rationally Designed Therapy. Oncologist. 2017 Jul;22(7):786-96.

22 Myall NJ, Neal JW, Cho-Phan CD, Zhou LY, Stehr H, Zhou L et al. Long-Term Survival of a Patient With Non-Small-Cell Lung Cancer Harboring a V600E Mutation in the BRAF Oncogene. Clin Lung Cancer. 2016 Mar;17(2):e17-21. 